\title{
Article \\ Maternal-Fetal Implications of SARS CoV-2 Infection during Pregnancy, Viral, Serological Analyses of Placenta and Cord Blood
}

\author{
Souhail Alouini ${ }^{1,2, * \mathbb{D}}$, Jerôme Guinard ${ }^{3}$, Olivier Belin ${ }^{4}$, Louis Mesnard ${ }^{1}$, Evelyne Werner ${ }^{5}$, Thierry Prazuck ${ }^{6}$ \\ and Chantal Pichon ${ }^{7}$
}

1 Department of Obstetrics and Gynecology, Centre Hospitalier Régional d'Orléans, 45100 Orléans, France; louis.mesnard@chr-orleans.fr

2 Faculté des Sciences, Université d'Orleans, 45100 Orleans, France

3 Department of Microbiology, Virology and Parasitology, Centre Hospitalier Régional d'Orléans, 45100 Orléans, France; jerome.guinard@chr-orleans.fr

4 Department of Anesthesiology and Reanimation, Centre Hospitalier Régional d'Orléans, 45100 Orléans, France; olivier.belin@chr-orleans.fr

5 Department of Pediatrics and Neonatal Intensive Unit Care, Centre Hospitalier Régional d'Orléans, 45100 Orléans, France; evelyne.werner@chr-orleans.fr

6 Department of Infectious and Tropical Diseases, Centre Hospitalier Régional d'Orléans, 45100 Orléans, France; thierry.prazuck@chr-orleans.fr

7 Centre de Biophysique Moléculaire, CNRS, Université d'Orléans, 45100 Orléans, France; chantal.pichon@univ-orleans.fr

* Correspondence: alouini.s@orange.fr or souhail.alouini@chr-orleans.fr; Tel.: +33-238613285

check for updates

Citation: Alouini, S.; Guinard, J.; Belin, O.; Mesnard, L.; Werner, E.; Prazuck, T.; Pichon, C. Maternal-Fetal Implications of SARS CoV-2 Infection during Pregnancy, Viral, Serological Analyses of Placenta and Cord Blood. Int. J. Environ. Res. Public Health 2022, 19, 2105. https://doi.org/10.3390/ ijerph19042105

Academic Editor: Paul B. Tchounwou

Received: 2 January 2022

Accepted: 10 February 2022

Published: 13 February 2022

Publisher's Note: MDPI stays neutral with regard to jurisdictional claims in published maps and institutional affiliations.

Copyright: () 2022 by the authors Licensee MDPI, Basel, Switzerland. This article is an open access article distributed under the terms and conditions of the Creative Commons Attribution (CC BY) license (https:// creativecommons.org/licenses/by/ $4.0 /)$.

\begin{abstract}
Objective: There are few data on the maternal-fetal transmission of SARS-CoV-2 and its outcomes. This study aimed to evaluate pregnancy outcomes of pregnant women infected by SARSCoV-2, to detect SARS-CoV-2 in placenta and different newborns' samples and search antibodies in cord blood. Methods: This was a prospective study of pregnant women diagnosed with SARS-CoV-2 infection from May 2020 to May 2021. At delivery, the placentas were investigated for SARS-CoV-2 using RT-PCR, cord blood. Mothers' blood samples were tested by SARS-CoV-2 serology. PCR of nasopharyngeal, anal and gastric swabs (NPSs) of newborns was performed according to pediatric indications. Results: Among 3626 pregnant women presenting at maternity to deliver, 45 mothers had COVID-19 during their pregnancy or at delivery (32 \pm 4.8 years). Most of them were multiparous and in the third trimester. There were 35 (77\%) women who remained in ambulatory, while $10(22 \%)$ were hospitalized for severe pneumonia, digestive symptoms, and/or fetal tachycardia. Thirty-eight delivered vaginally, and 7 had a cesarean delivery with normal Apgar scores ( $9 \pm 1.6$ at $5 \mathrm{~min}$ ) and umbilical artery $\mathrm{pH}(7.22 \pm 0.08)$. Two mothers required ICU admission after cesarean section for fetal and maternal distress. Of the 46 newborns, 6 were premature births (13\%) and 5 IUGR (intra-uterine growth restriction,11\%). RT-PCR SARS-CoV-2 was positive for $1 / 30$ placental, and 1/33 neonatal anal swabs and negative in all other cases and in gastric swabs. SARS-CoV-2 IgG was positive in 20/41 cord blood samples (49\%) and their mothers' samples. IgM was negative in the 23 cord blood samples. Conclusions: Pregnancy outcomes in women diagnosed with COVID-19 during their pregnancy were favorable in most cases. However, some women with severe clinical forms required hospitalization and ICU admission. Preterm births and intrauterine growth retardations were relatively frequent. Vaginal delivery was possible in most cases. SARS-CoV-2 IgG antibodies were positive and elevated in most cord blood samples of newborns. They are possibly of maternal origin, suggesting a probable mechanism of fetal protection against SARS-CoV-2 infection. No SARS-CoV-2 IgM was found in the cord blood samples. Detection of SARS-CoV-2 in placenta is rare.
\end{abstract}

Keywords: COVID-19; SARS-CoV-2; SARS-CoV-2 antibodies; coronavirus; cord blood; pregnancy; maternal-fetal transmission; real time-PCR; placenta 


\section{Introduction}

The COVID-19 pandemic is responsible for severe acute respiratory syndrome leading to thousands of deaths daily [1]. With almost no age spared, the SARS-CoV-2 infection has been reported in both pregnant and non-pregnant women equally [2,3]. However, the potential mechanism for in utero maternal-fetal transmission of the SARS-CoV-2 virus is uncertain and debatable. As newborns are also known to be infected by the SARS-CoV-2 virus [4], it is important to understand how the virus is transmitted in utero and/or in the postpartum period.

While some studies document that the SARS-CoV-2 was not recovered from the placenta or nasopharyngeal swabs of neonates born to COVID-19 positive mothers [2], a case of elevated SARS-CoV-2 IgM antibody in the neonate's blood at $2 \mathrm{~h}$ of life has been reported [5].

Establishing if there is in utero fetal transmission is important for identifying potential fetopathies that can be treated. In addition, unlike other viral infections (such as cytomegalovirus), it is still undefined whether the natural evolution of COVID-19 in pregnant women has serious consequences for the baby, both in utero and after birth [6-8]. Vivanti et al. [9] reported one case of maternal-fetal transmission of SARS-CoV-2. Thus, there is a need to ascertain the possibility of maternal-fetal transmission of COVID-19.

Pregnant women with COVID-19 commonly develop a moderate clinical form of the disease [4]. Likewise, there is a lack of evidence and evidence-based guidelines for fetal well-being and fetal outcomes in the case of maternal COVID-19 infection. In the previous studies reporting on COVID-19-positive pregnant women, the mode of delivery was mainly a cesarean section [2]. However, management of the newborns of mothers infected by COVID-19 remains problematic because of lack of evidence and guidelines in the domain.

Even though few data are available on SARS-CoV-2 serology in the cord blood and the maternal blood, the need for questioning on a possible passive-immunity mechanism in utero transferred by the mother to her fetus remains acute.

Based on these findings, we aimed to evaluate pregnancy outcomes of pregnant women infected by SARS-CoV-2, to detect SARS-CoV-2 in placenta and different newborns' samples and search antibodies in cord blood.

\section{Methods}

Pregnant women presenting for delivery at the Regional Hospital Center, Orleans, France, with COVID-19 infection from 5 May 2020 to 1 May 2021 were included in the study. The Maternity Department at the Regional Hospital Center, Orleans, France, is a tertiary referral center performing approximately 4900 deliveries per year, while the Hospital's Department of Infectious Diseases and the Laboratory of Virology are also referral departments for COVID-19. At the beginning of the COVID-19 pandemic in France, we prospectively elaborated a multidisciplinary protocol with infectiologists, virologists, pharmacologists, anesthesiologists, and gynecologists to manage pregnant women coming to the hospital who were suspected of COVID-19 infection

All pregnant women suspected of SARS-CoV-2 infection based on clinical signs (cough, fever, dyspnea, ageusia, anosmia, digestive symptoms or flu-like syndrome) had a polymerase chain reaction (RT-PCR) SARS-CoV-2 test on the nasopharyngeal swab and/or SARS-CoV-2 serology [10-12]. Only women who tested positive for SARS-CoV-2 who were between the ages of 18 and 49 years were included in the study. Written informed consent was obtained from each enrolled patient. S.A., O.B., and L.M. conducted the research. Only pregnant women with SARS-CoV-2 infection during their pregnancy or at delivery who came to the maternity for delivery were included in the study. Midwives, nurses, and doctors were involved in taking the nasopharyngeal swabs of the mothers and their newborns, anal and gastric swabs of the newborns, and of cord blood and placenta samples. All these samples were taken in the delivery room.

The study was approved by the CPP (Committee of Protection of Persons) Ouest II, France: number 2020-A01110-39. 


\section{Biological Exams}

The Real Time Polymerase chain reaction (RT-PCR) for SARS-CoV-2 was performed using the TaqPath multiplex COVID-19 kit (Thermo Fischer Scientifc, Waltham, MA, USA). All samples were processed at the Department of Virology, Regional Hospital Center of Orleans, France.

SARS-CoV-2 serology was performed with an immuno-enzymatic technique by chemiluminescence (Maglumi 800). The sensitivity of this technique is $95 \%$ at 15 days after the onset of clinical symptoms, and the specificity for IgM and IgG is $99 \%$ [13]. The results for SARS-CoV-2 serology were classified as follows:

For IgG: CoV-2 index negative if $<0.8$; positive if $>1.2$; and $0.8<$ gray zone $<1.2$.

For IgM: CoV-2 index negative if $<0.03$; positive if $>0.365$.

\section{Study Sample}

The sample consisted of pregnant women who were diagnosed with COVID-19 infection and consented to participate, and prospectively offered a placental and cord blood sample at delivery. RT- PCR for SARS-CoV-2 was done for the placental tissue, and SARSCoV-2 serology was performed on the cord blood. According to pediatric indications, anal and gastric samples of newborns were analyzed by PCR. In case of positive anal or gastric swabs for SARS-CoV-2 of the newborn, a PCR on vaginal and anal swabs of the mother was performed. In the case of respiratory or digestive clinical symptoms in the newborn or perinatal SARS-CoV-2 infection by the mother, a PCR on the neonate's nasopharyngeal swabs was performed, according to the pediatric indications. All pregnant women suspected of COVID-19 or with a history of COVID-19 during their pregnancy had serum examination for SARS-CoV-2 at the time of admission to the maternity center. All these samples were collected immediately after delivery in the operating room.

Besides the patients' demographic characteristics (age, BMI, parity, geographic origin), data for the number of weeks of gestation (WG), previous medical and obstetric history, comorbidities, clinical symptoms, the results for SARS-CoV-2 PCR and/or SARS-CoV-2 serology for pregnant women COVID-19 were recorded.

Severe COVID-19 was defined as dyspnea, respiratory rate of $\geq 30$ breaths per min, blood oxygen saturation $<94 \%$, lung infiltrates $>50 \%$, or need of mechanical ventilation or multiple organ failure $[14,15]$.

For fetal monitoring, the outcomes such as delivery mode, birth weight, Apgar scores, and umbilical artery $\mathrm{pH}$ were recorded for analysis. The following maternal outcomes were noted: hospital stay duration, follow-up for ambulatory patients, clinical and biological follow-up.

\section{Statistical Analysis}

Descriptive statistics, either absolute numbers and percentages for qualitative data or mean and standard deviations for quantitative data, were calculated. Followed by descriptive analyses using MS-Excel version 16.16.21 (Microsoft Corporation, Redmond, WA, USA).

\section{Results}

Among the 3626 patients presenting to the department of Obstetrics to deliver during the study period, 45 pregnant women (with a mean age of $32 \pm 4.8$ years) diagnosed with COVID-19 during their pregnancy and 46 newborns (1 twin pregnancy) were included in the study. Most of them were multiparous and in the third trimester of pregnancy (Table 1).

All pregnant women who came to the hospital for delivery were tested for COVID-19. The RT-PCR test on nasopharyngeal swabs was positive for all 45 patients, confirming the diagnosis of COVID-19 infection. No pregnant women with covid-19 were vaccinated during the study period. The major symptoms were fever, cough, ageusia, headache, dyspnea, anosmia, diarrhea or vomiting, and asthenia (Table 2). Most women were followed 
ambulatory. However, ten pregnant women (22\%) required hospital admission for major symptoms or in the case of fetal distress (Table 3 ).

Table 1. General characteristics of pregnant women with coronavirus disease (COVID-19).

\begin{tabular}{lcc}
\hline Characteristics & $\mathbf{N}$ & $\mathbf{\%}$ \\
\hline Age, mean \pm SD, years & $32 \pm 4.8$ & 68.89 \\
Multiparous & 31 & \\
Weeks of gestation & $31.14 \pm 8.25$ & \\
mean \pm SD at diagnosis & & 8.89 \\
Trimester of pregnancy with COVID-19 & 4 & 6.67 \\
1st trimester & 3 & 84.44 \\
2nd trimester & 38 & 80.00 \\
3rd Trimester & & 20.00 \\
Ethnic group & 36 & \\
White & 9 & 17.78 \\
Black & & 22.22 \\
Comorbidities & 8 & 8.89 \\
BMI (kg/m ${ }^{2}>30$ & 10 & 2.22 \\
Gestational diabetes & 4 & 2.22 \\
Hypothyroidism & 1 & 24.44 \\
Severe eczema & 1 & 2.22 \\
Asthma & 11 & 11.11 \\
Age $>35$ years & 1 & \\
Previous phlebitis & 5 & \\
Anemia $<8$ g Hb/dL & & \\
\hline
\end{tabular}

Table 2. Clinical symptoms of pregnant women diagnosed with COVID-19.

\begin{tabular}{lcc}
\hline Mother Characteristics & No. & Percent (\%) \\
\hline Clinical Symptoms COVID-19 $(\mathrm{n}=45)$ & 26 & 57.78 \\
\hline Cough & 11 & 24.44 \\
Fever & 17 & 37.78 \\
Dyspnea & 9 & 20.00 \\
Flu-like syndrome & 15 & 33.33 \\
Diarrhea/vomiting & 8 & 17.78 \\
Ageusia & 10 & 22.22 \\
Anosmia & 8 & 17.78 \\
Asymptomatic & 19 & 42.22 \\
\hline
\end{tabular}

Table 3. Diagnosis and outcomes of pregnant women with coronavirus disease (COVID-19).

\begin{tabular}{lcc}
\hline Mother Characteristics & No. & Percent (\%) \\
\hline COVID-19 Diagnosis of the Mother $(n=45)$ & & \\
\hline PCR NP swabs + & 45 & 700.00 \\
Followed ambulatory & 35 & 77.78 \\
Hospitalized & 10 & 22.22 \\
Pneumonia & 6 & 13.33 \\
Moderate & 3 & 6.67 \\
Severe & 3 & 6.67 \\
Pharmacotherapy & & \\
Paracetamol & 17 & 37.78 \\
Antiviral treatments & 4 & 8.89 \\
Heparin treatment & 15 & 0.30 \\
Oxygen therapy & 8 & 17.78 \\
Intensive unit care & 2 & 4.44 \\
\hline
\end{tabular}


Of the 45 women included in the study, 38 delivered vaginally (84.4\%) and 7 (15.6\%) by cesarean delivery (CD). Tenofovir was prescribed at the beginning of the study to 4 patients. Two women were admitted to the intensive care unit (ICU) and required mechanical ventilation for 30-45 days after cesarean delivery for severe COVID-19. The two patients were older than the others (age $>40$ years). One of them presented with severe anemia and the other with previous phlebitis and diabetes. Both also presented with severe pneumonia. The mean interval between the COVID-19 infection and delivery was $48 \pm 53$ days, varying from 0 to 220 days (Table 4 ). The mean umbilical artery $\mathrm{pH}$ was $7.22 \pm 0.08$, while Apgar scores were within normal limits $(9 \pm 1.6)$. There was one twin pregnancy. Of the 46 newborns, 6 were premature births (13\%), 5 IUGR (intra-uterine growth restriction, $11 \%$ ), and 5 had macrosomia (>90th percentile).

Table 4. Delivery of mothers COVID-19 and newborns outcomes.

\begin{tabular}{lcc}
\hline & Outcomes/N & Standard Deviation \\
\hline Mean day interval of COVID-19 delivery (days) & 48 & 53 \\
Term of birth (weeks of gestation) & 38.7 & 1.7 \\
Caesarean Delivery & 7 & \\
Vaginal delivery & 38 & 905.5 \\
Birth weight (g) & 3154.4 & 1.6 \\
Apgar score at $3 \mathrm{~mm}$ & 9.4 & 0.08 \\
Umbilical artery pH & 7.22 & \\
\hline
\end{tabular}

SARS-CoV-2 was detected by RT-PCR in 1(3.3\%) of the 30 placental samples at delivery (Table 5).

Table 5. SARS-CoV-2 findings at delivery in the placenta, cord blood, and newborns of mothers with COVID-19.

\begin{tabular}{lccc}
\hline PCR/Serology SARS-CoV-2 & Total & $\boldsymbol{n}$ Positive & Positive $\%$ \\
\hline IgG of mother at delivery & 37 & 20 & 54.05 \\
IgM SARS-CoV-2 of mother & 23 & 4 & 17.39 \\
PCR SARS-CoV-2 placenta & 30 & 1 & 3.33 \\
IgM cord blood & 23 & 0 & 0.00 \\
IgG cord blood & 41 & 20 & 48.78 \\
Anal newborn PCR swabs & 33 & 1 & 3.03 \\
Gastric NB PCR swabs & 33 & 0 & 0.00 \\
Nasopharyngeal newborn PCR & 13 & 0 & 0 \\
\hline
\end{tabular}

The PCR of the placenta did not detect SARS-CoV-2 in the 29 other cases.

SARS-CoV-2 was detected by RT-PCR in 1 (3\%) out of 33 anal swabs of newborns taken immediately after delivery. These results were verified by RT-PCR of the same samples after two days, which remained positive.

PCR was negative in the maternal vaginal and anal swabs. The RT-PCR on NPS of the newborn was also negative at day 2.

RT-PCR on newborn rectal and gastric samples was negative in all other cases (see Table 4), and all 13 NPSs of newborns suspected of COVID-19 were negative on RT-PCR and were healthy.

The IgG antibodies for SARS-CoV-2 were positive in 20 of the 41 cord blood samples (48.8\%) (Table 5), while IgM in the cord blood was negative for all cases (23/23). Likewise, IgG in the maternal blood was positive in 20 of the 37 cases $(54 \%)$ and IgM was positive in 4 of the 23 cases $(17 \%)$.

It was not possible to collect amniotic fluid samples because most women delivered vaginally and many of them had a premature or spontaneous rupture of membranes mixing the amniotic fluid with vaginal fluid. However, the rectal and gastric swabs were collected to reflect the amniotic status. 


\section{Discussion}

Forty-five mothers at delivery were included in this prospective study. The sample is considered small in clinical; however, it should be noted that clinics were not the main focus of the study. In the study period, we did not observe a high number of pregnant women infected by SARS-CoV-2 who delivered, even though our maternity is among the biggest in France. The aim of the study was more focused on the biological investigation of the maternal-fetal transmission of SARS-CoV-2 by analyzing cord blood and placental samples. Our study is prospective and one of the largest studies concerning the number of cord blood, placental samples, and anal and gastric swabs analyzed. Cosma et al. [16] analyzed 17 pregnant women who tested positive for COVID-19 and found that their newborns developed IgG antibodies. Colson et al. [17] analyzed 31 placentas of mothers who tested positive for COVID-19 and found one case of placental infection with SARS-CoV-2.

The 45 pregnant women with COVID-19 who participated in this study experienced clinical symptoms that varied greatly, from mildly symptomatic to severe forms that required hospitalization. COVID-19 was more easily detected in the last trimester of pregnancy; perhaps the infection was less symptomatic in the first trimester of pregnancy and thus less susceptible to detection. Most patients were managed symptomatically and were given ambulatory care. Those who were hospitalized developed more severe symptoms of COVID-19 and experienced acute respiratory signs, such as moderate-tosevere pneumonia, with or without digestive symptoms of diarrhea, vomiting, and nausea. Ellington et al. [2] found that pregnant women were likelier to be hospitalized and require mechanical ventilation compared to non-pregnant women. As for the present study, only two patients needed mechanical ventilation. Our study showed that COVID-19 in pregnant women manifested mainly in benign forms, even though some patients needed mechanical ventilation, a result that differed from initial studies, which reported that COVID-19 was merely a benign disease in pregnant women. Despite the low number of patients hospitalized in the ICU, the findings are in accordance with other studies, which highlighted that the majority of pregnant women COVID-19 experienced minor or moderate forms of the disease. [18].

Most pregnant women with COVID-19 delivered a healthy newborn at term vaginally. Premature birth was frequent, and CD was indicated for $15 \%$ of women. Previous reports [2] have documented cesarean delivery as most frequently used in COVID-19 patients. In the current study, vaginal delivery has proven to be safe and should be emphasized for pregnant women with COVID-19.

In our study, SARS-CoV-2 was detected in one placental sample and one anal swab of a neonate (born to a mother with a history of COVID-19 during pregnancy) taken immediately after delivery. RT PCR SARS-CoV-2 of NP swabs and anal swabs of their mothers were negative at delivery. Vivanti et al. [9] reported a case of maternal-fetal transmission of SARS-CoV-2. They detected the virus in the placenta. Hence, the presence of SARS-CoV-2 in the placental and anal samples of a newborn immediately at birth suggests maternal-fetal transmission of SARS-CoV-2 during pregnancy or a maternal contamination of samples at delivery.

According to Shah et al. [19], when SARS CoV-2 is not found in the cord blood and the placenta, the maternal-fetal transmission is unlikely. However, Gong et al. [20] reported positive anal swabs in 10 children, even with negative throat swabs. Feco-oral transmission is also a probable route for SARS-CoV-2 infection as suggested by Yuan et al. [20], who found SARS CoV-2 in anal swabs of children with COVID-19 infection.

It is noteworthy that Chen et al. [4] did not find viral RNA in 3 placental tissues obtained from women with COVID-19 infection. Similarly, Fan et al. [8] reported that SARSCoV-2 was not detected by PCR in two products of conception and infants. In the same way, Chen et al. [2] have reported that the amniotic fluid and cord blood in 6 infected patients was negative for SARS-CoV-2 on PCR. However, the placenta, anal, and gastric samples of the newborns were not tested, and no serodiagnosis of SARS-CoV-2 was performed on 
the cord blood [2] in the placenta, anal and gastric, and nasopharyngeal samples of the neonates born to COVID-19-positive mothers.

In this study, SARS-CoV-2 IgG antibody levels were elevated in cord blood samples at delivery, as well as in mothers' blood. The presence of IgG in the cord blood of many newborns is probably due to the passage of maternal IgG antibodies in the cord blood. These antibodies are probably protective against COVID-19 infection in the fetus and may explain why the fetus is rarely infected by the virus. In many cases, the SARS-CoV-2 IgG serology was negative in both of the aforementioned samples. In these cases, the mother was asymptomatic, indicative of a low viral charge. In addition, many COVID-19 mothers did not develop IgM and IgG antibodies even in the presence of a positive PCR of their nasopharyngeal swabs. This is probably due to low viral-plasmatic circulation.

Another important finding was that we did not discover SARS-CoV-2 IgM antibodies in all cord blood samples tested, even though the sensitivity and specificity of the immune-enzymatic technique used were excellent at $95 \%$ and $99 \%$, respectively. However, Dong et al. [5] reported one case of elevated IgM antibodies at two hours of life in a newborn.

\section{Limitations and Strengths of the Study}

First, the sample size was relatively small; however, this prospective investigation is among the largest studies that have evaluated the maternal-fetal transmission of SARSCoV-2. The study includes prospective analyses and the results of placental, cord blood samples, and anal, gastric, and nasopharyngeal swabs of newborns and COVID-19 mothers at delivery.

Second, not all samples could be tested, as our hospital was overwhelmed by the COVID-19 waves. At times, the samples did not arrive at the laboratory in good condition. In other cases, the blood samples were hemolyzed; it was then impossible to perform viral analysis.

In addition, our study's strength is that it demonstrated that vaginal delivery of healthy newborns of mothers with COVID-19 is possible in the majority of cases; this contrasts with the findings of other studies, wherein caesarean section deliveries were performed in most cases [2].

Our study is among the largest studies to have prospectively analyzed (contrary to many retrospective studies) placenta and cord blood samples. It showed that pregnant women with COVID-19 transmitted SARS-CoV-2 IgG antibodies to their fetuses via cord blood to protect them in utero against an acute fetal viral infection. These findings provide a probable explanation for why very few fetuses were infected in utero by SARS-CoV-2 and are correlated with the fact that we did not find SARS-CoV-2 IgM in the analyzed cord blood samples. Indeed, the presence of IgM in the blood signifies an acute infection in utero. Other authors found IgM in the cord blood in one case [5]. Thirty placentas were analyzed prospectively for SARS-CoV-2 at delivery. This sample is among the largest studies that have analyzed the presence of the virus in the placenta. It showed one case of a placenta positive for SARS-CoV-2, which suggests in utero SARS-CoV-2 transmission. This study confirms the results of other studies: maternal-fetal transmission of SARS-CoV-2 is possible, but it remains rare.

The analysis of both placental and cord blood samples explains why in utero viral infection is rare.

\section{Conclusions}

In this prospective study, most women recovered well with symptomatic management. However, patients with severe clinical forms required hospitalization or ICU admission. There were preterm births and intrauterine growth restrictions in pregnancies complicated by COVID-19. Pregnant women with COVID-19 should be carefully monitored clinically and by ultrasonography in order to diagnose these pathologies. 
Vaginal delivery was possible and should be recommended for the majority of pregnant women with COVID-19.

Our study showed that pregnant women with COVID-19 transmitted the SARS-CoV$2 \mathrm{Ig} G$ antibodies to their fetuses via the cord blood. These findings could be a very probable explanation of why very few fetuses are infected in utero by SARS-CoV-2 and are correlated to the fact that we did not find the SARS-CoV-2 IgM in the analyzed cord blood samples. These findings suggest a probable mechanism of fetal protection and passive fetal immunization against SARS-CoV-2 infection.

We found one case of positive placenta for SARS-CoV-2, which suggests in utero SARS-CoV-2 transmission. Future larger studies are highlighted to confirm our results.

Author Contributions: Conceptualization, S.A., J.G., O.B., L.M. and C.P.; methodology, S.A. and J.G., O.B. and L.M.; validation, S.A., J.G., L.M., O.B., E.W., T.P. and C.P.; formal analysis, S.A., J.G., O.B. and L.M.; investigation, S.A., J.G., L.M., O.B., E.W. and T.P.; resources, S.A.; data curation, S.A.; writing-original draft preparation, S.A.; writing-review and editing, S.A.; visualization, S.A. and J.G.; supervision, S.A., L.M. and O.B.; project administration, S.A. and C.P. All authors have read and agreed to the published version of the manuscript.

Funding: This research received no external funding.

Institutional Review Board Statement: The study was approved by the CPP (Committee of Protection of Persons) Ouest II, France, number 2020-A01110-39.

Informed Consent Statement: Informed consent was obtained for all subjects involved in the study.

Data Availability Statement: Data are available from the corresponding author under reasonable request.

Acknowledgments: Thanks to Emilie Colin, Midwife Responsible in the Maternity of Orleans for helping to organize the collection of samples and to all Doctors and Midwifes who participated to the collection of samples.

Conflicts of Interest: The authors declare no conflict of interest.

\section{References}

1. COVID-19 Situation Update Worldwide. Available online: https://www.ecdc.europa.eu/en/geographical-distribution-2019 -ncov-cases. (accessed on 10 February 2022).

2. Che, H.; Guo, J.; Wang, C.; Luo, F.; Yu, X.; Zhang, W.; Li, J.; Zhao, D.; Xu, D.; Gong, Q.; et al. Clinical characteristics and intrauterine vertical transmission potential of COVID-19 infection in nine pregnant women: A retrospective review of medical records. Lancet 2020, 395, 809-815, Correction in Lancet 2020, 395, 1038. [CrossRef]

3. Han, R.; Huang, L.; Jiang, H.; Dong, J.; Peng, H.; Zhang, D. Early Clinical and CT Manifestations of Coronavirus Disease 2019 (COVID-19) Pneumonia. AJR Am. J. Roentgenol. 2020, 215, 1-6. [CrossRef] [PubMed]

4. Wong, Y.P.; Khong, T.Y.; Tan, G.C. The Effects of COVID-19 on Placenta and Pregnancy: What Do We Know So Far? Diagnostics 2021, 11, 94. [CrossRef] [PubMed]

5. $\quad$ Dong, L.; Tian, J.; He, S.; Zhu, C.; Wang, J.; Liu, C.; Yang, J. Possible Vertical Transmission of SARS-CoV-2 From an Infected Mother to Her Newborn. JAMA 2020, 323, 1846-1848. [CrossRef] [PubMed]

6. Chen, S.; Huang, B.; Luo, D.J.; Li, X.; Yang, F.; Zhao, Y.; Nie, X.; Huang, B.X. Pregnancy with new coronavirus infection: Clinical characteristics and placental pathological analysis of three cases. Zhonghua Bing Li Xue Za Zhi 2020, 49, 418-423. [CrossRef] [PubMed]

7. Stagno, S.; Pass, R.F.; Cloud, G.; Britt, W.J.; Henderson, R.E.; Walton, P.D.; Veren, D.A.; Page, F.; Alford, C.A. Primary cytomegalovirus infection in pregnancy. Incidence, transmission to fetus, and clinical outcome. JAMA 1986, 256, $1904-1908$. [CrossRef] [PubMed]

8. Yow, M.D.; Williamson, D.W.; Leeds, L.J.; Thompson, P.; Woodward, R.M.; Walmus, B.F.; Lester, J.W.; Six, H.R.; Griffiths, P.D. Epidemiologic characteristics of cytomegalovirus infection in mothers and their infants. Am. J. Obstet. Gynecol. 1988, 158, $1189-1195$. [CrossRef]

9. Fan, C.; Lei, D.; Fang, C.; Li, C.; Wang, M.; Liu, Y.; Bao, Y.; Sun, Y.; Huang, J.; Guo, Y.; et al. Perinatal Transmission of COVID-19 Associated SARS-CoV-2: Should We Worry? Clin. Infect. Dis. 2021, 72, 862-864. [CrossRef] [PubMed]

10. Vivanti, A.J.; Vauloup-Fellous, C.; Prevot, S.; Zupan, V.; Suffee, C.; Cao, J.D.; Benachi, A.; De Luca, D. Transplacental transmission of SARS-CoV-2 infection. Nat Commun. 2020, 11, 3572. [CrossRef] [PubMed]

11. Wang, W.; Xu, Y.; Gao, R.; Lu, R.; Han, K.; Wu, G.; Tan, W. Detection of SARS-CoV-2 in different types of clinical specimens. JAMA 2020, 323, 1843-1844. [CrossRef] [PubMed] 
12. Woo, P.C.; La, S.K.; Wong, B.H.; Tsoi, H.-w.; Fung, A.M.Y.; Chan, K.-h.; Tam, V.K.P.; Malik Peiris, J.S.; Yuen, K.-y. Detection of specific antibodies to severe acute respiratory syndrome (SARS) coronavirus nucleocapsid protein for serodiagnosis of SARS coronavirus pneumonia. J. Clin. Microbiol. 2004, 42, 2306-2309. [CrossRef] [PubMed]

13. Xu, W.; Li, J.; He, X.; Zhang, C.; Mei, S.; Li, C.; Li, Y.; Cheng, S.; Zhang, P. The diagnostic value of joint detection of serum IgM and IgG antibodies to 2019-nCoV in 2019-nCoV infection. Chin. J. Lab. Med. 2020, 43, E012.

14. Van Tol, S.; Mögling, R.; Li, W.; Godeke, G.-J.; Swart, A.; Bergmans, B.; Brandenburg, A.; Kremer, K.; Murk, J.-L.; van Beek, J.; et al. Accurate Serology for SARS-CoV-2 and common Human Coronaviruses using a Multiplex Approach. Emerg. Microbes Infect. 2020, 9, 1965-1973. [CrossRef] [PubMed]

15. The Novel Coronavirus Pneumonia Emergency Response Epidemiology Team. The epidemiological characteristics of an outbreak of 2019 novel coronavirus diseases (COVID-19)_China, 2020. China CDC Wkly. 2020, 2, 113-122. [CrossRef]

16. Cosma, S.; Carosso, A.R.; Corcione, S.; Cusato, J.; Borella, F.; Antonucci, M.; Marozio, L.; Revelli, A.; Preti, M.; Ghisetti, V.; et al. Longitudinal analysis of antibody response following SARS-CoV-2 infection in pregnancy: From the first trimester to delivery. J. Reprod. Immunol. 2021, 144, 103285. [CrossRef] [PubMed]

17. Colson, A.; Depoix, C.L.; Dessilly, G.; Baldin, P.; Danhaive, O.; Hubinont, C.; Sonveaux, P.; Debiève, F. Clinical and in Vitro Evidence against Placenta Infection at Term by Severe Acute Respiratory Syndrome Coronavirus 2. Am. J. Pathol. 2021, 191, 1610-1623. [CrossRef] [PubMed]

18. Shah, P.S.; Diambomba, Y.; Acharya, G.; Morris, S.K.; Bitnun, A. Classification system and case definition for SARS-CoV-2 infection in pregnant women, fetuses, and neonates. Acta Obstet. Gynecol. Scand. 2020, 99, 565-568. [CrossRef] [PubMed]

19. Xu, Y.; Li, X.F.; Zhu, B.; Liang, H.; Fang, C.; Gong, Y.; Guo, Q.; Sin, X.; Zhao, D.; Shen, J.; et al. Characteristics of pediatric SARS-CoV-2 infection and potential evidence for persistent fecal viral shedding. Nat. Med. 2020, 26, 502-505. [CrossRef] [PubMed]

20. Yuan, C.; Zhu, H.; Yang, Y.; Cai, X.; Xiang, F.; Wu, H.; Yao, C.; Ziang, Y.; Xiao, H. Viral loads in throat and anal swabs in children infected with SARS-CoV-2. Emerg. Microbes Infect. 2020, 9, 1233-1237. [CrossRef] [PubMed] 